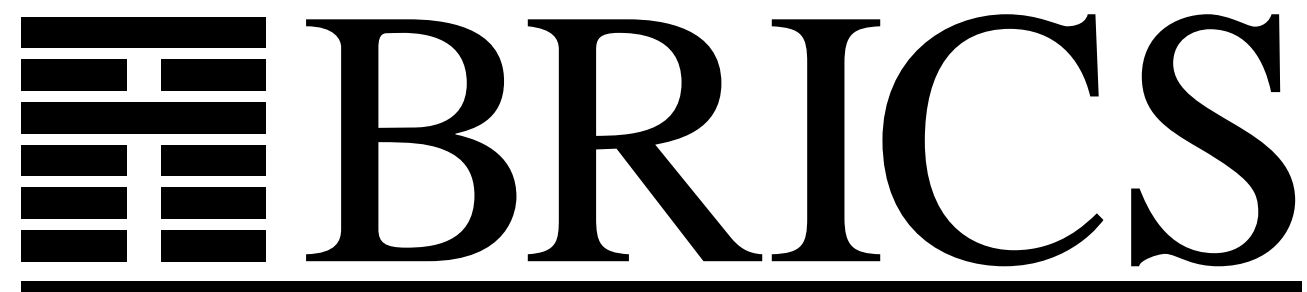

Basic Research in Computer Science

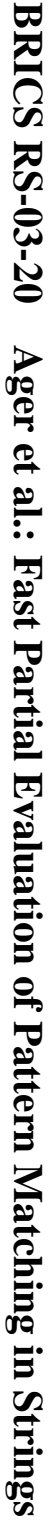

\title{
Fast Partial Evaluation of Pattern Matching in Strings
}

Mads Sig Ager

Olivier Danvy

Henning Korsholm Rohde

BRICS Report Series

RS-03-20 
Copyright (c) 2003, Mads Sig Ager \& Olivier Danvy \&

Henning Korsholm Rohde.

BRICS, Department of Computer Science

University of Aarhus. All rights reserved.

Reproduction of all or part of this work is permitted for educational or research use on condition that this copyright notice is included in any copy.

See back inner page for a list of recent BRICS Report Series publications. Copies may be obtained by contacting:

\author{
BRICS \\ Department of Computer Science \\ University of Aarhus \\ Ny Munkegade, building 540 \\ DK-8000 Aarhus C \\ Denmark \\ Telephone: +45 89423360 \\ Telefax: $\quad+4589423255$ \\ Internet: BRICS@brics.dk
}

BRICS publications are in general accessible through the World Wide Web and anonymous FTP through these URLs:

http: //www.brics.dk

ftp: / / ftp.brics.dk

This document in subdirectory RS / 03/20/ 


\title{
Fast Partial Evaluation of Pattern Matching in Strings *
}

\author{
Mads Sig Ager, Olivier Danvy, and Henning Korsholm Rohde \\ BRICS $^{\dagger}$ \\ Department of Computer Science \\ University of Aarhus ${ }^{\ddagger}$
}

April 2003

\begin{abstract}
We show how to obtain all of Knuth, Morris, and Pratt's linear-time string matcher by partial evaluation of a quadratic-time string matcher with respect to a pattern string. Although it has been known for 15 years how to obtain this linear matcher by partial evaluation of a quadratic one, how to obtain it in linear time has remained an open problem.

Obtaining a linear matcher by partial evaluation of a quadratic one is achieved by performing its backtracking at specialization time and memoizing its results. We show (1) how to rewrite the source matcher such that its static intermediate computations can be shared at specialization time and (2) how to extend the memoization capabilities of a partial evaluator to static functions. Such an extended partial evaluator, if its memoization is implemented efficiently, specializes the rewritten source matcher in linear time.
\end{abstract}

\footnotetext{
* To appear in the proceedings of the ACM SIGPLAN 2003 Workshop on Partial Evaluation and Semantics Based Program Manipulation (PEPM), San Diego, California, June 7, 2003.

${ }^{\dagger}$ Basic Research in Computer Science (www.brics.dk),

funded by the Danish National Research Foundation.

$¥$ Ny Munkegade, Building 540, DK-8000 Aarhus C, Denmark.

E-mail: \{mads, danvy, hense\}@brics.dk
} 


\section{Contents}

1 Introduction $\quad 3$

2 Obtaining a specialized matcher that works in linear time $\quad 3$

3 Linear-time specialization $\mathbf{5}$

3.1 Compositional backtracking ............. 6

3.2 Strengthening the memoization capabilities of the specializer . . 7

3.3 Specializing the staged matcher in linear time . . . . . . . . 8

4 From Morris-Pratt to Knuth-Morris-Pratt 9

5 Related work $\quad 10$

6 Conclusion and perspectives 10

$\begin{array}{ll}\text { A Partial evaluation of staged string matchers } & 11\end{array}$

\section{List of Figures}

1 A staged quadratic-time string matcher . . . . . . . . . . . 4

2 Sharing of computations with compositional backtracking . . . . 6

3 Compositional backtracking suitable for fast partial evaluation . 8

4 Backtracking also using one character of negative information . . 9

5 A brute-force quadratic-time string matcher . . . . . . . . . . . . 12

6 A specialized linear-time matcher . . . . . . . . . . . . . 13 


\section{Introduction}

For 15 years now, it has been a traditional exercise in partial evaluation to obtain Knuth, Morris, and Pratt's string matcher by specializing a quadratictime string matcher with respect to a pattern string [11,21]. Given a quadratic string matcher that searches for the first occurrence of a pattern in a text, a partial evaluator specializes this string matcher with respect to a pattern and yields a residual program that traverses the text in linear time. The problem was first stated by Yoshihiko Futamura in 1987 [15] and since then, it has given rise to a variety of solutions $[2,3,10,13,14,15,16,19,25,28,29,32,33]$.

For 15 years, however, it has also been pointed out that the solutions only solve half of the problem. Indeed, the Knuth-Morris-Pratt matcher first produces a 'next' table in time linear in the length of the pattern and then traverses the text in time linear in the length of the text. In contrast, a partial evaluator does not specialize a string matcher in linear time. This shortcoming was already stated in Consel and Danvy's first report of a solution [10] and it has been mentioned ever since, up to and including Futamura's keynote speech at ASIA-PEPM 2002 [13].

In this article, we solve the remaining half of the problem.

Prerequisites: We expect a passing familiarity with partial evaluation and string matching as can be gathered in Jones, Gomard, and Sestoft's textbook [21] or in Consel and Danvy's tutorial notes [11]. In addition, we distinguish between the Knuth-Morris-Pratt matcher and the Morris-Pratt matcher in that the former uses one character of negative information whereas the latter does not [7]. Our string matchers are expressed in a first-order subset of the Scheme programming language [23]. They are specialized using polyvariant program-point specialization [27], where certain source program points (specialization points) are indexed with static values and kept in a 'seen-before' list (i.e., memoized), and residual program points are mutually recursive functions.

In the rest of this article, we use the terms "partial evaluator" and "(program) specializer" interchangeably.

\section{Obtaining a specialized matcher that works in linear time}

The essence of obtaining a linear-time string matcher by partial evaluation of a quadratic-time string matcher is to ensure that backtracking is carried out at specialization time. To obtain this effect, one can either rewrite the matcher so that backtracking only depends on static data (such a rewriting is known as a binding-time improvement or a staging transformation [27]) and use a simple partial evaluator $[4,10]$, or keep the matcher as is and use a sophisticated partial evaluator $[13,29,32]$. In this article, the starting point is a staged quadratictime matcher and a simple memoizing partial evaluator, such as Similix, where specialization points are dynamic conditionals or dynamic functions [6]. 


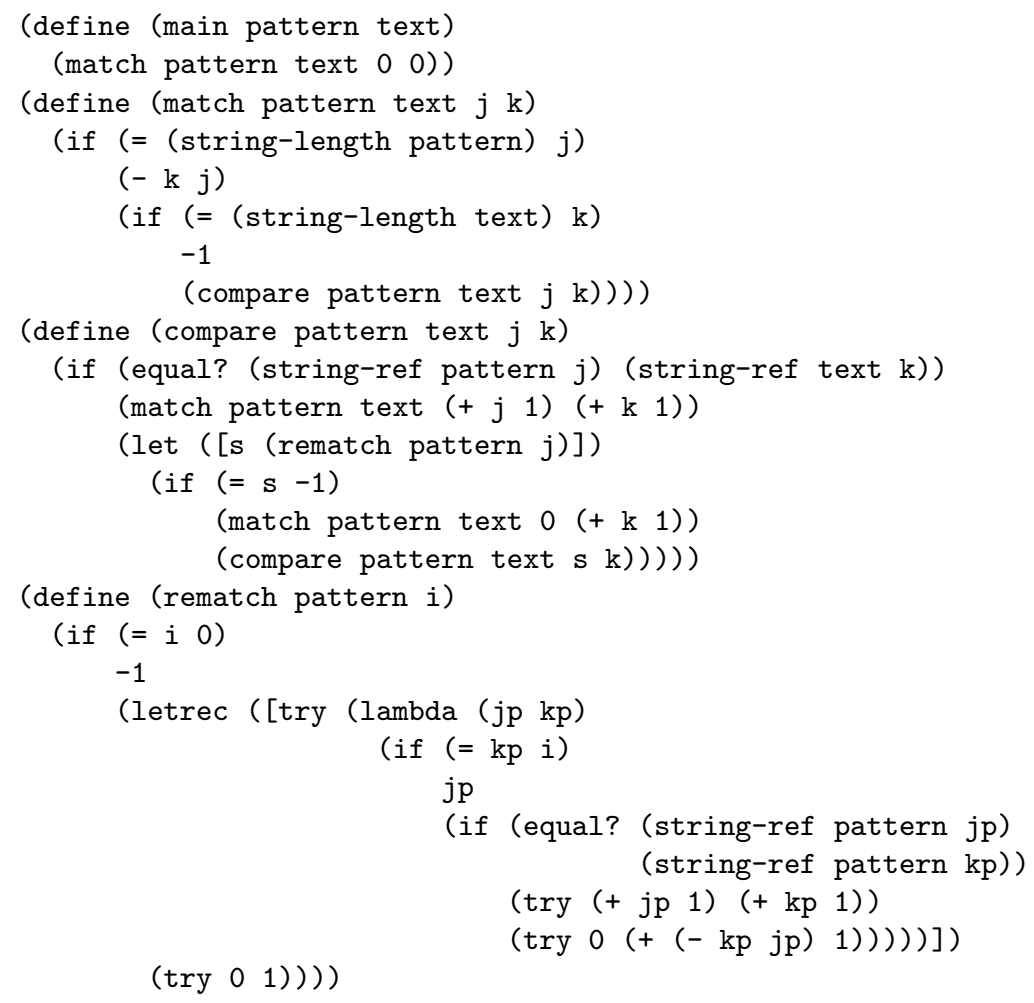

Figure 1: A staged quadratic-time string matcher

- main is the matcher's entry point which directly calls match.

- match checks whether matching should terminate, either because an occurrence of the pattern has been found in the text or because the end of the text has been reached. If not, compare is called to perform the next character comparison. For simplicity, we assume that string-length works in constant time; otherwise, we would compute the lengths once and pass them as parameters.

- compare checks whether the $j$ th character of the pattern matches the kth character of the text. If so, match is called to match the rest of the pattern against the rest of the text. If not, rematch is called to backtrack based on the part of the pattern that did match the text.

- rematch backtracks based on a part of the pattern. It returns an index corresponding to the length of the longest proper prefix that is also a suffix of the given part of the pattern. If such a prefix does not exist it returns -1 . The returned index corresponds to the index returned by the Morris-Pratt failure function [1]. The local recursive function try finds the length of the longest proper prefix of the pattern that is also a suffix by successively trying each proper prefix. 
Figure 1 displays a staged matcher similar to the ones developed in the literature $[1,4,10,21]$ (see Appendix A for a more complete picture). Matching is done naively from left to right. After a mismatch the pattern is shifted one position to the right and matching resumes at the beginning of the pattern. Since we know that a prefix of the pattern matches a part of the text, we use this knowledge to continue matching using the pattern only. This part of matching performs backtracking and is done by the rematch function. The matcher is staged because backtracking only depends on static data. The key to linear-time string matching is that backtracking can be precomputed either into a lookup table as in the Morris-Pratt matcher or into a residual program as in partial evaluation.

If a specializer meets certain requirements, specializing the matcher of Figure 1 with respect to a pattern string yields a linear-time matcher that behaves like the Morris-Pratt matcher. Specifically, the specializer must compute static operations at specialization time and generate a residual program where dynamic operations do not disappear, are not duplicated, and are executed in the same order as in the source program.

\section{Linear-time specialization}

As already shown in the literature [1, 17], each specialized version of a staged matcher such as that of Figure 1 has size linear in the length of the pattern. For two reasons, however, specialization does not proceed in time linear in the length of the pattern. The first reason is that for every position in the pattern, the specializer blindly performs the backtracking steps of the staged quadratic matcher. These backtracking steps are carried out by static functions which are not memoization points and whose results are not memoized. But even if the results were memoized, the backtracking steps would still be considered unrelated because of the index that caused the mismatch. The second reason is connected to an internal data structure of the specializer. Managing the seen-before list, which is really a dictionary, as a list is simply not fast enough.

In order to achieve linear-time specialization the matcher must be rewritten such that the backtracking steps become related, the memoization capabilities of the specializer must be extended to handle static functions, and the implementation of the memoization must be efficient.

Terminology: For the purpose of analysis, static backtracking is a mathematical function that takes a string - a problem - and returns a (possibly empty) prefix of that string - the solution - such that the solution is the longest proper prefix of the problem that is also a suffix of the problem. A subproblem is a prefix of a problem. A computation is the computational steps involved in applying static backtracking to a given problem. Given a pattern, backtracking at position $i$ is the computation where the problem is the prefix of length $i$ of the pattern. 


\subsection{Compositional backtracking}

We relate backtracking at different positions by expressing the backtracking compositionally, i.e., by expressing a solution to a problem in terms of solutions to its subproblems. Backtracking is performed by the rematch function and we rewrite it so that it becomes recursive and unaware of its context (thus avoiding continuations or the index that originally caused a mismatch).

Figure 2 illustrates how to express backtracking compositionally and how it enables sharing of intermediate computations at specialization time. For the pattern abacababb, the backtracking at positions 3,7 , and 8 are the computations

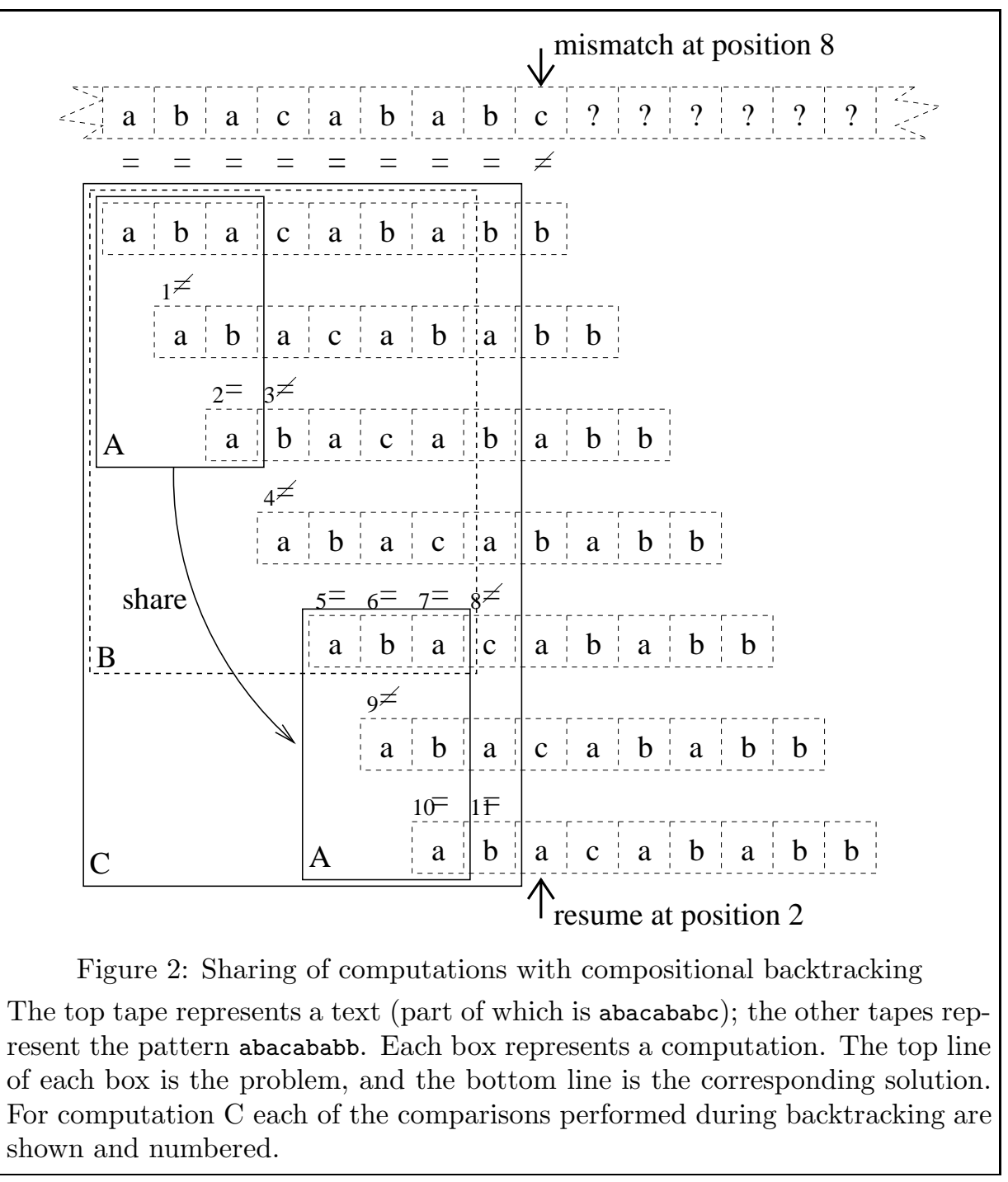


marked with A, B, and C, respectively. In general, backtracking at position i is always the first part of backtracking at position $i+1$, and ideally the solution to the first computation can directly be extended to a solution to the second one.

Let us consider what to do if the solution cannot be extended. The solution given by computation $\mathrm{B}$, aba, is an example of this, since comparison 8 fails and therefore abac is not the solution to computation $\mathrm{C}$. However, the solution aba is by definition the longest proper prefix of abacaba that is also a suffix. Since the solution aba is a prefix, it is also a subproblem, namely the problem of computation A, and since it is a suffix, part of the continued backtracking (comparisons 9 and 10) is identical to computation A. Computation A can therefore be shared. In the same manner as before, we try to extend the solution given by computation $\mathrm{A}$, a, to the solution to computation $\mathrm{C}$. In this case the solution can be extended to ab.

In short, the key observation is that the solution given by computation $\mathrm{B}$ is equal to the problem in computation $\mathrm{A}$, and therefore computation $\mathrm{A}$ can be shared within computation C. The solution to static backtracking on a given problem can therefore be expressed in terms of solutions to static backtracking on subproblems.

By expressing backtracking compositionally, we obtain the staged matcher displayed in Figure 3, which is suitable for fast partial evaluation. The rematch function has been rewritten to use a local recursive function, try-subproblem, that tries to extend the solutions to subproblems to a full solution. The backtracking part of the matcher now allows sharing of computations.

\subsection{Strengthening the memoization capabilities of the spe- cializer}

Despite the further rewriting, the specializer is still not able to exploit the compositional backtracking. The reason is that the specializer only memoizes at specialization points. Since specialization points are dynamic conditionals or dynamic functions, and the recursive backtracking is purely static, the specializer does not memoize the results.

What is needed is static memoization, where purely static program points are memoized and used within the specialization process itself. The results of purely static functions should be cached and used statically to avoid redoing past work. In the partial evaluator, static memoization is then essentially the same as the usual-dynamic-memoization. As usual with tabulation techniques, the requirements imposed on both types of memoization are that initialization, insertion and retrieval can be done in constant time (amortized). For the string matchers presented in this article these requirements can be met by a dictionary that uses a (growing) hash-table and a collision-free hash function based on the pattern and the index into the pattern. To avoid rehashing the pattern at all memoization points, we must remember hash values for static data. In general, more advanced hashing mechanisms would be needed and the time complexities of initialization, insertion and retrieval would be weakened from constant time to expected constant time. 


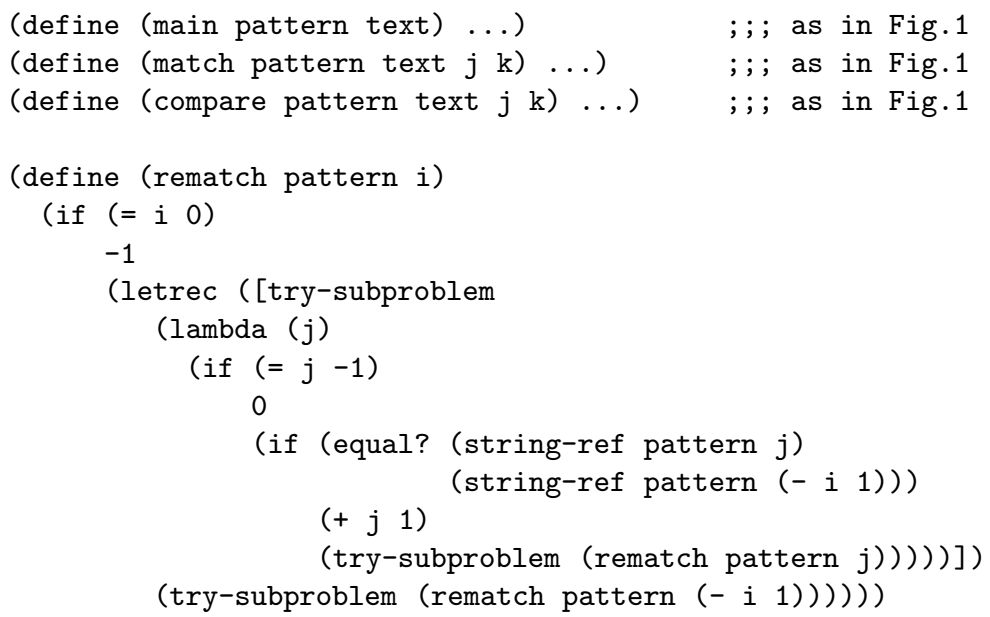

Figure 3: Compositional backtracking suitable for fast partial evaluation

Compared to Figure 1, rematch has been rewritten to allow sharing of computations. It calls itself recursively to find the solution to the immediate subproblem. The local recursive function try-subproblem then tries to extend the solution to the immediate subproblem to a solution to the original problem. If the solution cannot be extended, rematch is called to find the next candidate solution.

\subsection{Specializing the staged matcher in linear time}

Given these efficient memoization capabilities, the rewritten matcher can be specialized in linear time. Each of the linear number of residual versions of compare and match can clearly be generated in constant time. We therefore only have to consider specializing the rematch function.

Since rematch always calls itself recursively on the immediate subproblem and all results are memoized, we only need to ensure that backtracking with respect to the largest problem, i.e., backtracking at position $i$, where $i$ is the length of the pattern, is done in linear time. Recursive calls and returns take linear time. For a given subproblem at $j$, however, try-subproblem may be unfolded up to $j$ times. Unfolding only occurs more than once if the solution to the subproblem cannot be extended to a full solution, that is, if the $j-1$ st character causes a mismatch. Therefore, the additional time spent in all calls to try-subproblem is proportional to the overall number of mismatches during backtracking. Since backtracking is just (staged) brute-force string matching, the number of mismatches is clearly no greater than the length of the pattern.

Generating the residual versions of the rematch function can therefore also be done in linear time and the entire specialization process takes linear time. 


\section{From Morris-Pratt to Knuth-Morris-Pratt}

The Morris-Pratt matcher and the Knuth-Morris-Pratt matcher differ in that the latter additionally uses one character of negative information [7]. Therefore, the Knuth-Morris-Pratt matcher statically avoids repeated identical mismatches by ensuring that the character at the resume position is not the same as the character at the mismatch position.

Extending the result to the Knuth-Morris-Pratt matcher is not difficult. The only caveat is that we cannot readily use backtracking at position $i$ in backtracking at position $i+1$, because with negative information the solution at $i$ is never a part of the solution at $i+1$. Instead, we observe that the solution to the simpler form of backtracking where the negative information is omittedMorris-Pratt backtracking - is indeed always a part of the solution.

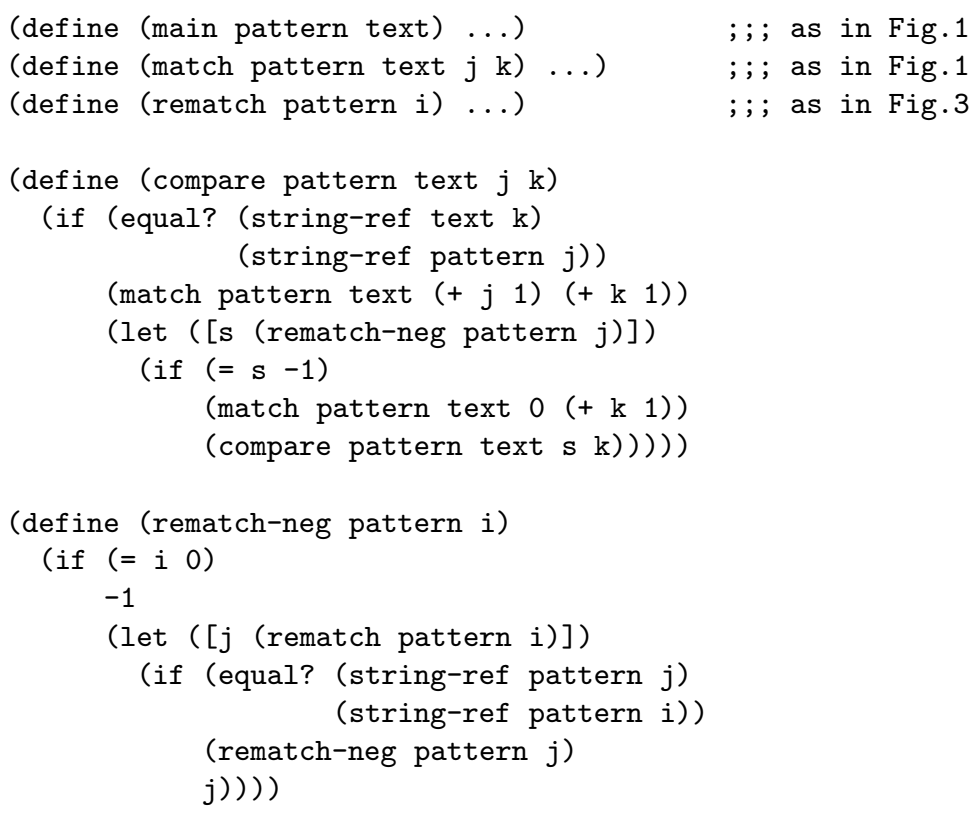

Figure 4: Backtracking also using one character of negative information

Compared to Figure 3, a wrapper function rematch-neg for rematch has been added. compare calls rematch-neg instead of rematch. rematch-neg calls rematch to compute the solution to Morris-Pratt backtracking. It then checks whether this solution is also a solution to Knuth-Morris-Pratt backtracking by performing an extra character comparison. If the solution to Morris-Pratt backtracking is not a solution to Knuth-Morris-Pratt backtracking (the character comparison fails) rematch-neg calls itself recursively to compute the solution. 
The matcher in Figure 4 uses this observation. Based on Morris-Pratt backtracking as embodied in the rematch function of Figure 3, the rematch-neg function computes the solution to Knuth-Morris-Pratt backtracking. If both rematch and rematch-neg are statically memoized, evaluating them for all positions at specialization time can be done in linear time.

\section{Related work}

The Knuth-Morris-Pratt matcher has been reconstructed many times in the program-transformation community since Knuth's own construction (he obtained it by calculating it from Cook's construction [24, page 338]). Examples of the methods used are Dijkstra's invariants [12], Bird's recursion introduction and tabulation [5], Takeichi and Akama's equational reasoning [33], Colussi's Hoare logic [9], and Hernández and Rosenblueth's logic-program derivation [18].

Bird's recursion introduction and tabulation is our closest related work. Bird derives the Morris-Pratt matcher from a quadratic time stack algorithm using recursion introduction. The recursive failure function he derives is essentially the same as the rematch function of Figure 3. Bird then tabulates the failure function to obtain the linear time preprocessing phase of the Morris-Pratt matcher.

Takeichi and Akama's equational reasoning is our second closest related work. By hand (i.e., without using a partial evaluator), they transform a quadratic-time functional string matcher into the linear-time Morris-Pratt matcher. As part of the transformation, they isolate a function equivalent to the MorrisPratt failure function. Using partial parameterization and memoization data structures, this function is tabulated in time linear in the size of a pattern string, thereby obtaining the Morris-Pratt matcher.

\section{Conclusion and perspectives}

We have shown how to obtain all of Knuth, Morris, and Pratt's linear-time string matcher by partial evaluation of a quadratic-time string matcher with respect to a pattern string. Obtaining a linear-time string matcher by partial evaluation was already known, but obtaining it in linear time was an open problem.

To this end, we have rewritten the staged matcher so that its backtracking is compositional, thereby enabling sharing of computations at specialization time. We have also identified that the sharing of dynamic computations as achieved with the traditional seen-before list [21] is not enough; static computations must also be shared. The concepts involved - staging, i.e., binding-time separation, and sharing of computations as in dynamic programming - have long been recognized as key ones in partial evaluation $[3,26]$. They are, however, not sufficient to obtain linear-time string matchers in linear time. In addition, the static computations must be shared by memoization, and both the static and the dynamic memoization mechanisms must be efficient. Static memoization in itself is no silver bullet: a program must be (re)written so that static computations can 
be shared-which still requires some degree of insight from the programmer; otherwise, as usual with tabulation techniques, memoization is just a waste of resources.

Independently of partial evaluation, we can also consider the staged matchers by themselves. To this end, we can express them as functional programs with memo-functions, i.e., in some sense, as fully lazy functional programs. These programs, given efficient memoization capabilities, are the lazy-functional equivalent of the Morris-Pratt and Knuth-Morris-Pratt imperative matchers. (Holst and Gomard as well as Kaneko and Takeichi made a similar observation [19, 22].) In particular, these programs work in linear time.

Finally, we would like to point out that the Knuth-Morris-Pratt matcher is not an end in itself. Fifteen years ago, this example was used to show that partial evaluators needed considerable power (be it polyvariant program-point specialization or generalized partial computation) to produce efficient specialized programs. It gave rise to the so-called KMP test [30, 31]. What our work shows today is that a partial evaluator needs even more power (static memoization and efficient data structures) to operate efficiently. Such an efficient partial evaluator remains to be implemented.

Acknowledgments: We are grateful to Julia Lawall for many useful comments. This article has also benefited from the anonymous reviewers' comments. This work is supported by the ESPRIT Working Group APPSEM II (http://www . appsem.org).

\section{A Partial evaluation of staged string matchers}

We provide a more complete view of partial evaluation of staged string matchers (see also Section 2). The fundamental starting point is always a brute-force quadratic-time string matcher, such as the one in Figure 5. The corresponding ending point is always a specialized linear-time string matcher, such as the one in Figure 6, which is specialized to match the pattern abac. Here, the specialized matcher behaves exactly like the Morris-Pratt matcher for the pattern abac.

As it happens, specializing the brute-force quadratic matcher using a simple memoizing partial evaluator, such as Similix, does not yield a linear-time matcher (only a linear-sized one). One way to overcome this problem is to rewrite or stage the quadratic matcher such that backtracking only depends on static data and therefore can be computed by the partial evaluator. However, partial evaluation then takes quadratic time, because the overall workload has not been reduced-only moved.

This work describes how to actually reduce the workload by further rewriting the staged matcher (Figure 3) and by enhancing the memoization capabilities of the partial evaluator (see Section 3 ). 


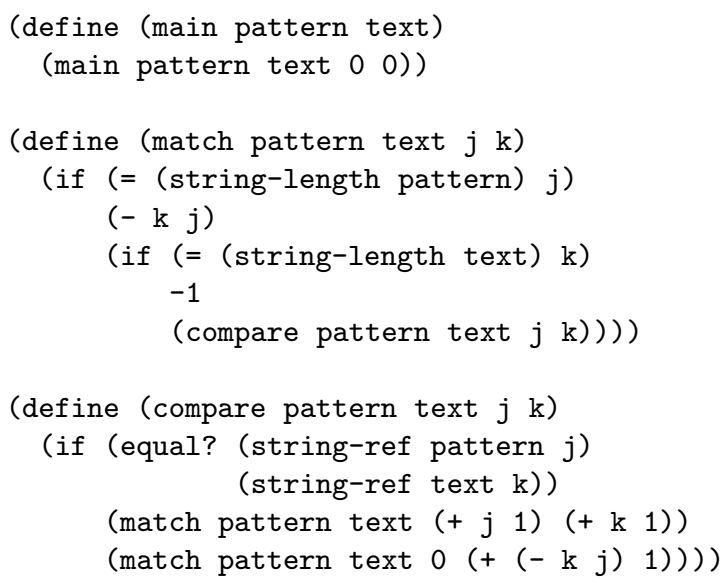

Figure 5: A brute-force quadratic-time string matcher

- main is the matcher's entry point which directly calls match.

- match checks whether matching should terminate, either because an occurrence of the pattern has been found in the text or because the end of the text has been reached. If not, compare is called to perform the next character comparison. Again, for simplicity, we assume that string-length works in constant time.

- compare checks whether the jth character of the pattern matches the $\mathrm{kth}$ character of the text. If so, match is called to match the rest of the pattern against the rest of the text. If not, the whole pattern and the corresponding part of the text are restored, the text position is incremented by one character, and match is called to restart matching.

Pictorially, the situation is as follows:

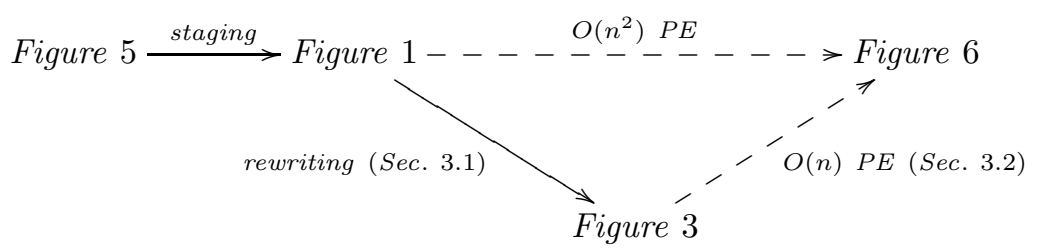

The matchers in Figures 1, 3, and 5 perform the exact same sequence of character comparisons; they differ only in where they fetch the text characters during backtracking. 


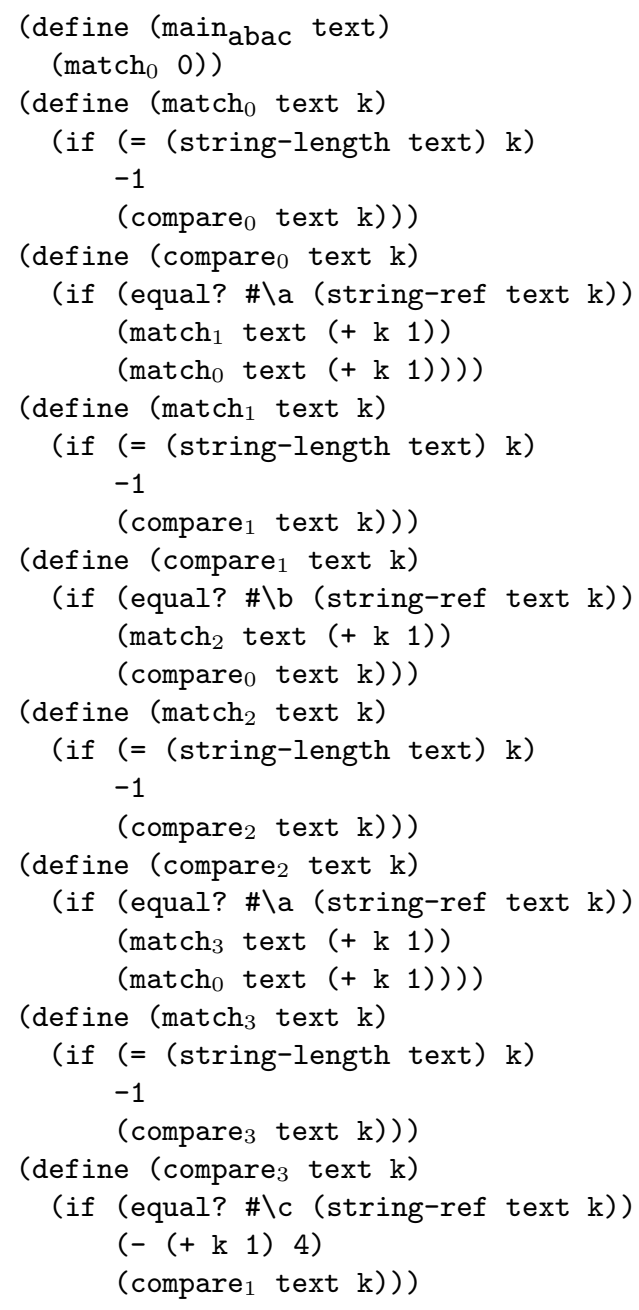

Figure 6: A specialized linear-time matcher

The connection to the matchers in Figures 1, 3, and 5 is:

- For all strings text,

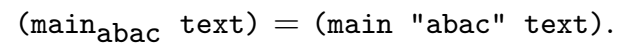

- For all strings text, integers k, and $i \in\{0,1,2,3\}$, $\left(\right.$ match $_{i}$ text $\left.\mathrm{k}\right)=($ match "abac" text $i \mathrm{k})$.

- For all strings text, integers $\mathbf{k}$, and $i \in\{0,1,2,3\}$, ( compare $_{i}$ text $\left.\mathrm{k}\right)=($ compare "abac" text $i \mathrm{k})$. 


\section{References}

[1] Mads Sig Ager, Olivier Danvy, and Henning Korsholm Rohde. On obtaining Knuth, Morris, and Pratt's string matcher by partial evaluation. In Chin [8], pages 32-46. Extended version available as the technical report BRICSRS-02-32.

[2] Maria Alpuente, Moreno Falaschi, Pascual Juliàn, and German Vidal. Specialization of inductively sequential functional logic programs. In Charles Consel, editor, Proceedings of the ACM SIGPLAN Symposium on Partial Evaluation and Semantics-Based Program Manipulation, pages 151-162, Amsterdam, The Netherlands, June 1997. ACM Press.

[3] Torben Amtoft. Sharing of Computations. PhD thesis, DAIMI, Department of Computer Science, University of Aarhus, 1993. Technical report PB-453.

[4] Torben Amtoft, Charles Consel, Olivier Danvy, and Karoline Malmkjær. The abstraction and instantiation of string-matching programs. In Torben Æ. Mogensen, David A. Schmidt, and I. Hal Sudborough, editors, The Essence of Computation: Complexity, Analysis, Transformation. Essays Dedicated to Neil D. Jones, number 2566 in Lecture Notes in Computer Science, pages 332-357. Springer-Verlag, 2002. Extended version available as the technical report BRICS RS-01-12.

[5] Richard S. Bird. Improving programs by the introduction of recursion. Communications of the ACM, 20(11):856-863, November 1977.

[6] Anders Bondorf. Similix 5.1 manual. Technical report, DIKU, Computer Science Department, University of Copenhagen, Copenhagen, Denmark, May 1993. Included in the Similix 5.1 distribution.

[7] Christian Charras and Thierry Lecroq. Exact string matching algorithms. http://www-igm.univ-mlv.fr/ lecroq/string/, 1997.

[8] Wei-Ngan Chin, editor. ACM SIGPLAN Asian Symposium on Partial Evaluation and Semantics-Based Program Manipulation, Aizu, Japan, September 2002. ACM Press.

[9] Livio Colussi. Correctness and efficiency of pattern matching algorithms. Information and Computation, 95:225-251, 1991.

[10] Charles Consel and Olivier Danvy. Partial evaluation of pattern matching in strings. Information Processing Letters, 30(2):79-86, January 1989.

[11] Charles Consel and Olivier Danvy. Tutorial notes on partial evaluation. In Susan L. Graham, editor, Proceedings of the Twentieth Annual ACM Symposium on Principles of Programming Languages, pages 493-501, Charleston, South Carolina, January 1993. ACM Press.

[12] Edsger W. Dijkstra. A Discipline of Programming. Prentice-Hall, 1976. 
[13] Yoshihiko Futamura, Zenjiro Konishi, and Robert Glück. Automatic generation of efficient string matching algorithms by generalized partial computation. In Chin [8], pages 1-8.

[14] Yoshihiko Futamura, Zenjiro Konishi, and Robert Glück. Program transformation system based on generalized partial computation. New Generation Computing, 20(1):75-99, 2002.

[15] Yoshihiko Futamura and Kenroku Nogi. Generalized partial computation. In Dines Bjørner, Andrei P. Ershov, and Neil D. Jones, editors, Partial Evaluation and Mixed Computation, pages 133-151. North-Holland, 1988.

[16] Robert Glück and Andrei Klimov. Occam's razor in metacomputation: the notion of a perfect process tree. In Patrick Cousot, Moreno Falaschi, Gilberto Filé, and Antoine Rauzy, editors, Proceedings of the Third International Workshop on Static Analysis WSA'93, number 724 in Lecture Notes in Computer Science, pages 112-123, Padova, Italy, September 1993. Springer-Verlag.

[17] Bernd Grobauer and Julia L. Lawall. Partial evaluation of pattern matching in strings, revisited. Nordic Journal of Computing, 8(4):437-462, 2002.

[18] Manuel Hernández and David A. Rosenblueth. Development reuse and the logic program derivation of two string-matching algorithms. In Harald Søndergaard, editor, Proceedings of the Third International Conference on Principles and Practice of Declarative Programming, pages 38-48, Firenze, Italy, September 2001. ACM Press.

[19] Carsten K. Holst and Carsten K. Gomard. Partial evaluation is fuller laziness. In Hudak and Jones [20], pages 223-233.

[20] Paul Hudak and Neil D. Jones, editors. ACM SIGPLAN Symposium on Partial Evaluation and Semantics-Based Program Manipulation, SIGPLAN Notices, Vol. 26, No 9, New Haven, Connecticut, June 1991. ACM Press.

[21] Neil D. Jones, Carsten K. Gomard, and Peter Sestoft. Partial Evaluation and Automatic Program Generation. Prentice-Hall International, London, UK, 1993. Available online at http://www.dina.kvl.dk/ sestoft/pebook/.

[22] Keiichi Kaneko and Masato Takeichi. Derivation of a Knuth-Morris-Pratt algorithm by fully lazy partial computation. Advances in Software Science and Technology, 5:11-24, 1993.

[23] Richard Kelsey, William Clinger, and Jonathan Rees, editors. Revised ${ }^{5}$ report on the algorithmic language Scheme. Higher-Order and Symbolic Computation, 11(1):7-105, 1998.

[24] Donald E. Knuth, James H. Morris, and Vaughan R. Pratt. Fast pattern matching in strings. SIAM Journal on Computing, 6(2):323-350, 1977. 
[25] Laura Lafave and John P. Gallagher. Constraint-based partial evaluation of rewriting-based functional logic programs. In Norbert E. Fuchs, editor, 7th International Workshop on Program Synthesis and Transformation, number 1463 in Lecture Notes in Computer Science, pages 168-188, Leuven, Belgium, July 1997. Springer-Verlag.

[26] Torben Æ. Mogensen. Binding Time Aspects of Partial Evaluation. PhD thesis, DIKU, Computer Science Department, University of Copenhagen, Copenhagen, Denmark, March 1989.

[27] Torben Æ. Mogensen. Glossary for partial evaluation and related topics. Higher-Order and Symbolic Computation, 13(4):355-368, 2000.

[28] Christian Queinnec and Jean-Marie Geffroy. Partial evaluation applied to pattern matching with intelligent backtrack. In Proceedings of the Second International Workshop on Static Analysis WSA'92, volume 81-82 of Bigre Journal, pages 109-117, Bordeaux, France, September 1992. IRISA, Rennes, France.

[29] Donald A. Smith. Partial evaluation of pattern matching in constraint logic programming languages. In Hudak and Jones [20], pages 62-71.

[30] Morten Heine Sørensen. Turchin's supercompiler revisited. an operational theory of positive information propagation. Master's thesis, DIKU, Computer Science Department, University of Copenhagen, April 1994. DIKU Rapport 94/17.

[31] Morten Heine Sørensen, Robert Glück, and Neil Jones. Towards unifying partial evaluation, deforestation, supercompilation, and GPC. In Donald Sannella, editor, Proceedings of the Fifth European Symposium on Programming, number 788 in Lecture Notes in Computer Science, pages 485-500, Edinburgh, Scotland, April 1994. Springer-Verlag.

[32] Morten Heine Sørensen, Robert Glück, and Neil D. Jones. A positive supercompiler. Journal of Functional Programming, 6(6):811-838, 1996.

[33] Masato Takeichi and Yoji Akama. Deriving a functional Knuth-MorrisPratt algorithm. Journal of Information Processing, 13(4):522-528, 1990. 


\section{Recent BRICS Report Series Publications}

RS-03-20 Mads Sig Ager, Olivier Danvy, and Henning Korsholm Rohde. Fast Partial Evaluation of Pattern Matching in Strings. May 2003. 16 pp. Final version to appear in Leuschel, editor, ACM SIGPLAN Workshop on Partial Evaluation and SemanticsBased Program Manipulation, PEPM '03 Proceedings, 2003. This report supersedes the earlier BRICS report RS-03-11.

RS-03-19 Christian Kirkegaard, Anders Møller, and Michael I. Schwartzbach. Static Analysis of XML Transformations in Java. May 2003. 29 pp.

RS-03-18 Bartek Klin and Pawel Sobociński. Syntactic Formats for Free: An Abstract Approach to Process Equivalence. April 2003. 41 pp.

RS-03-17 Luca Aceto, Jens Alsted Hansen, Anna Ingólfsdóttir, Jacob Johnsen, and John Knudsen. The Complexity of Checking Consistency of Pedigree Information and Related Problems. March 2003. 31 pp. This paper supersedes BRICS Report RS-02-42.

RS-03-16 Ivan B. Damgård and Mads J. Jurik. A Length-Flexible Threshold Cryptosystem with Applications. March 2003. 19 pp.

RS-03-15 Anna Ingólfsdóttir. A Semantic Theory for Value-Passing Processes Based on the Late Approach. March 2003. 48 pp.

RS-03-14 Mads Sig Ager, Dariusz Biernacki, Olivier Danvy, and Jan Midtgaard. From Interpreter to Compiler and Virtual Machine: A Functional Derivation. March 2003. 36 pp.

RS-03-13 Mads Sig Ager, Dariusz Biernacki, Olivier Danvy, and Jan Midtgaard. A Functional Correspondence between Evaluators and Abstract Machines. March 2003. 28 pp.

RS-03-12 Mircea-Dan Hernest and Ulrich Kohlenbach. A Complexity Analysis of Functional Interpretations. February 2003. 70 pp.

RS-03-11 Mads Sig Ager, Olivier Danvy, and Henning Korsholm Rohde. Fast Partial Evaluation of Pattern Matching in Strings. February 2003. 14 pp. This report is superseded by the later report BRICS RS-03-20.

RS-03-10 Federico Crazzolara and Giuseppe Milicia. Wireless Authentication in $\chi$-Spaces. February 2003. 20 pp. 